\title{
Distribution of Artificial Thrombi Candidates Through Patient-Specific Aortic-Arch Phantoms under Pulsatile Flow Conditions
}

Marco Testaguzza ( $\square$ marco.testaguzza@umons.ac.be)

University of Mons (UMONS)

Mehdi Benhassine

University of Mons (UMONS)

Haroun Frid

FridMind Technologies

Laurence Gebhart

FridMind Technologies

Karim Zouaoui Boudjeltia

Université libre de Bruxelles, $\mathrm{CHU}$ de Charleroi

Axel Vanrossomme

$\mathrm{CHU}$ de Charleroi

Gregory Coussement

University of Mons (UMONS)

\section{Research Article}

Keywords: Ischemic Stroke, emboli, pulsatile flow conditions

Posted Date: April 28th, 2021

DOl: https://doi.org/10.21203/rs.3.rs-455601/v1

License: (9) (i) This work is licensed under a Creative Commons Attribution 4.0 International License.

Read Full License 


\section{Abstract}

Ischemic Stroke is the most frequent type of stroke and is subject to many studies investigating prevention means. Avoiding the difficulties and ethical problems of experimental in-vivo research, in-vitro testing is a convenient way of studying in controlled conditions the morphological impact and mechanical aspects of emboli dynamics. This in-vitro study was performed with two realistic silicone aortic-arch phantoms submitted to physiological pulsatile flow conditions. In the in-vitro test bed, using automatic image tracking and analysis, it was made possible detecting and tracking artificial spherical emboli candidates circulating in the anatomic aortic-arch models under a realistic based-patient blood flow profile. The emboli trajectories as well as their repartition in the different supra-aortic branches are presented for the two aortic-arch geometries obtained from CT scans. Through a statistical analysis performed with several artificial emboli sizes, the experimental study shows that the repartition percentages of the emboli closely follow the flowrate repartition percentages for both aortic-arch models, suggesting that higher flowrates lead to higher concentrations of emboli in a given artery. Sets of human thrombi were also injected and the repartition percentages have been established, giving the same trend as for artificial emboli.

\section{Background And Purpose}

Over 13.7 million people experience a stroke event each year, out of which 9.5 million are ischemic strokes [1]. Several risk factors are known to increase the propensity of ischemic strokes such as atrial fibrillation and diabetes. According to Malone et al [2], the embolus size is not the only responsible factor for stroke propensity as the flowrate may also impact the number of emboli which can be recovered in arteries.

Some computational studies of emboli dynamics are available [3], [4], [5]. Choi's study focused on the comparison between atrial fibrillation and normal heart flow with emboli in the size range of 2 to 6 millimeters. Fabbri [4] considers micrometric particles but for a study on the Circle of Willis and does not consider trajectories in the prior part of the anatomy such as the aortic arch where flowrates are more elevated. Experimental works are either considering small spherical polyamide particles in the Circle of Willis [6] or millimetric thrombi analogues made from bovine blood within the aortic arch [2], [7].

Cardiogenic emboli sizes can range from milliliters to centimeters, depending on flow conditions, but emboli as small as a few hundred microns can be responsible for ischemic stroke depending on flow conditions especially in small cerebral capillaries [8]. A critical information is how geometry and shape (diameter and position) of the aortic arch and arteries affect the probability of emboli passage. The arteries discussed are the brachio-cephalic artery (BCA), the left-common-carotid artery (LCCA) and the left subclavian artery (LSA), out of which only one division branch of the BCA (the right common carotid artery) and the LCCA lead to the brain. The stroke propensity is shown to be directly linked to the diameter of the arteries in Choi's study [3] but only with the atrial fibrillation flow. The purpose of this study is to explore, in-vitro, such assumptions, at first with the normal flow. Carr [9] provides simulations and 
cardiogenic emboli distributions in the aortic arch and shows that, on average, particles in the size from 910 microns to 1920 microns have twice the likelihood of cardiac output to the arteries leading to the brain. Lately, Malone presented results indicating that blood clots made from bovine blood followed the flowrate percentages of atrial fibrillation pulsatile signals for aortic-arch phantoms made of silicone with artificial blood liquid as a medium [10].

Based on patients 4D-MRI scanners, two aortic arch geometries were extracted, and two patient-specific silicone models were created. A non-pathological pulsatile flowrate was reproduced in an in-vitro chamber simulating a vertically standing patient. The pulsatile flowrate signal imposed in the ascending aorta corresponds to the measurement on a real patient and the downstream flowrates repartition follows physiological values from the literature [11]. Artificial spherical polyethylene particles of different sizes, from 250 microns to 1 millimeter, were injected into the ascending aorta (AAO) and the trajectories were recorded using two video cameras. A computer vision algorithm was employed to detect and count the passage of the emboli out of the aortic arch through an analysis performed on several thousands of artificial emboli. To study the statistical repartition of the emboli in the aortic arch geometry, spherical particles were used to remove any shape related effects and provide repeatability like in the study design of Bushi [12]. To make sure that the choice of artificial emboli is relevant, verification experiments are made with thrombi made from human blood and the repartition results are compared.

\section{Materials And Method}

\section{In-Vitro Test-Bed Setup}

The system setup and the in-vitro test bed are illustrated on Fig. 1. It is vertically structured to account for gravity effects, thus offering a better representation of physiological conditions as it is considered that an average person spends 8 hours sleeping and 16 hours either sitting or standing [13].

The aortic arch model is placed at eye-level and branched at each exit, namely: ascending and descending aortas (AAO and DAO), BCA, LCCA and LSA. To record the instantaneous flowrate at each exit, flowmeters (Sonotec C0.55/230HV2.0 and C0.55/140V2.0) are employed while solenoid valves (Bürkert 8605 controller and type 2836 valves) allow tweaking the initial flowrate repartitions. Pressure sensors (First Sensor CTE8001GY4N and CTEM8500GY4N) are placed at each exit to monitor instantaneous pressure. To reproduce the flowrate, a UMONS patented system of pulsatile pump consisting of a centrifugal rotative pump in series with a piston pump has been exploited to reproduce physiological flow conditions [14]. Distilled water was chosen in this study as the difference with blood provides minimal differences in the aortic arch dynamics (the non-newtonian behavior is assumed to be negligible considering the diameter of the main arteries) and for its convenience and optic properties. Future works will consider artificial blood.

\section{Aortic Arch Model}


A set of imaging data was provided by the André Vésale Hospital (CHU de Charleroi, Belgium). Data included patient aortic arch 3D geometries comprising ascending (AAO) and descending (DAO) aortas, brachio-cephalic artery (BCA), left common carotid artery (LCCA) and the subclavian artery (LSA), extracted from computed tomography angiograms (angio-CT) as well as flow-velocity profile and AAO surface area changes over the cardiac cycle at the ascending aorta, quantified by phase contrast MRI. The first geometry (named model 1) came from a patient having suffered a stroke event while the second geometry (named model 2) came from a non-pathological patient. The open-source software for medical image informatics 3DSlicer (https://www.slicer.org/) [15] was used to smooth and clean the 3D chest scanners to extract the final aortic-arch geometry in each case. The three upper arteries final sections were modified for fixation purposes within the in-vitro chamber system using Autodesk Inventor.

The models were then printed in 3D using the open-source software CURA with a polyvinyl-acetate watersoluble filament (Ultimaker PVA). The model was used as a mold to generate the respective negative cast. Clear Sylgard 184 silicone elastomer and the hardener (Sigma Aldrich) were used for the molding process. The procedure to create the models included creating vacuum for degassing and the models were cured for 48 hours. Upon polymerization, the PVA was dissolved in flowing warm water until full dissolution of the print occurred. Figure 2 displays the final silicone models as well as the stages towards their creation.

The two geometries were characterized as follows: Model 1 and 2 had the following dimensions measured (three measurements were made on the STL files at the entrance in the middle and on the upper part of each artery):

Table 1 Aortic-arch models' dimensions, AAO is the ascending aorta, BCA is the BrachioCephalic artery, LCCA the left common carotid artery, LSA the subclavian artery and DAO is the descending aorta. Error bars were determined by taking three measurements at the base, middle and top parts of each section.

\begin{tabular}{|l|c|c|c|c|c|}
\hline Diameter $(\mathrm{mm})$ & AAO & BCA & LCCA & LSA & DAO \\
\hline Model 1 & 27 & $11.4 \pm 0.4$ & $5.9 \pm 0.3$ & $11.3 \pm 0.4$ & 20.5 \\
\hline Model 2 & 27 & $9.2 \pm 1.1$ & $6.43 \pm 0.7$ & $9.81 \pm 2.2$ & 20.5 \\
\hline
\end{tabular}

\section{Physiological Flow Reproduction}

\section{Non-Pathological Signal Reproduction}

The AAO pulsatile flowrate was measured at André Vésale Hospital. The patient's aorta diameter was measured at $34.2 \mathrm{~mm}$ resulting in an area of $9.210^{-4} \mathrm{~m}^{2}$. The silicone model created has an area of 5.72 $10^{-4} \mathrm{~m}^{2}$ and is molded on a different patient, therefore the flowrate at the ascending aorta had to be scaled down by $37.8 \%$. 
A UMONS patented system of pumps was exploited to reproduce the behavior of the physiological pulsatile flowrate profile [14]. The technology consisted of a centrifugal pump (Brushless DC Motor Water Pump DKB60TSA 24V) and a piston pump driven by a linear actuator. A membrane was fixed directly to the piston head to maintain a sealed and low friction coupling with a PMMA cylinder. The centrifugal pump was controlled by a NI-myRIO (National Instruments) together with the NI-LabView 2018 software and set to provide the mean aortic flowrate value. The linear actuator software (LinMot-Talk v6.6) was programmed to set the piston to follow a specific displacement profile in order to reproduce the imposed reference physiological signal.

The imposed piston motion allowed for the geometry adapted ascending aorta flow to be set (Fig. 3). The in-vitro chamber mean flowrate were measured with the associated standard deviation presented in Table 2.

Table 2 Mean flowrate measurements as well as repartition with respect to AAO and standard deviation for the 2 models as measured by the test-bed.

\begin{tabular}{|l|c|c|c|c|}
\hline & \multicolumn{2}{|c|}{ Model 1 } & \multicolumn{2}{c|}{ Model 2 } \\
\hline Artery & Flow [1/min] & Repartition [\%] & Flow [1/min] & Repartition [\%] \\
\hline AAO & $4.22 \pm 0.04$ & 100 & $4.11 \pm 0.41$ & 100 \\
\hline BCA & $0.85 \pm 0.01$ & $20.04 \pm 0.31$ & $0.86 \pm 0.03$ & $20.87 \pm 2.36$ \\
\hline LCCA & $0.71 \pm 0.02$ & $16.91 \pm 0.21$ & $0.71 \pm 0.04$ & $17.25 \pm 1.19$ \\
\hline LSA & $0.77 \pm 0.01$ & $18.33 \pm 0.29$ & $0.83 \pm 0.03$ & $20.10 \pm 2.10$ \\
\hline DAO & $1.84 \pm 0.02$ & $42.60 \pm 0.15$ & $1.75 \pm 0.31$ & $42.60 \pm 4.10$ \\
\hline
\end{tabular}

These values remain in the range of physiologically acceptable range [7]. The difference in the repartitions for the two models can be attributed to the fact that the arteries diameters are different but AAO signals remained the same as it is a model constraint to have imposed a constant inlet diameter.

The following graph (Fig. 4) presents a 10-seconds excerpt of the test-bed flowrates for the 5 branches.

\section{Artificial Thrombi Particles}

For optical efficiency in particle tracking, fluorescent green spherical particles made of polyethylene were used as artificial emboli candidates (obtained from Cospheric L.L.C, Santa Barbara, USA). The particle sizes used in this study were 250-325, 355-425, 600-710 and 850-1000 microns (each particle set has a dispersion documented by the manufacturer). The size values represent a continuous range to explore size effects. The density of the spheres was $1.025 \mathrm{~g} / \mathrm{cc}$. This density choice was justified as to retain a mass density close to human thrombi $(1.06 \mathrm{~g} / \mathrm{cc})$ [16] and satisfy optimal optical conditions for detection (fluorescent coating). The relative density between water and particles achieved buoyancy and remained close to the density ratio between blood and a blood clot made from platelets ( 1067/1050) [17]. A 
solution of polyethylene particles was prepared by mixing distilled water with Tween20 surfactant solution from Cospheric $(0.2 \%)$. The surfactant solution was used to avoid particles from aggregating and to reduce sticking in the tube and silicone models.

\section{Human Thrombi}

Human microthrombi were formed from whole blood taken from a volunteer in a tube without anticoagulant. After $30 \mathrm{~min}$, at $37^{\circ} \mathrm{C}$, the tube was centrifuged at $4000 \mathrm{~g}$ for 10 minutes to remove residual serum. A scalpel was used to cut thrombi of uniform size.

\section{Particles Injection Method}

Polyethylene particles were injected via a syringe equipped with a needle ( $19 \mathrm{G}$ and $22 \mathrm{G}$ needles) into a three-way valve to avoid any air being injected into the test-bed. A small magnet was put inside of the syringe and an agitator allowed to stir the tween-distilled water-polyethylene particles solution. The filled container was injected slowly and in a continuous manner to avoid as much as possible multiple particles entering at the same time.

\section{Imaging}

Trajectories of the emboli were recorded at 180 FPS (with the variable frame rate setting with 100MBPS 8Bit Full HD resolution) using two Panasonic Lumix GH5 cameras with Olympus M.Zuiko Macro 60mm lenses. One camera was mounted on a Z-tilt mount on the Rexroth Chassis while the other one was fixed on a Genesis A3 tripod. One camera recorded only the BCA, LCCA and LSA branches whereas the other camera recorded a portion of the transparent tube connected with the ascending Aorta (AAO) into which particles were injected with a syringe. A polarized diaphragm was affixed to the macro lens for the camera fixed on the tripod to improve contrast and to filter unwanted light reflections. For this camera, the GH5 settings were ISO 400, F 11, shutter $1 / 1600$ s, a portrait mode and a custom white balance setting which rendered the best possible contrast for the green particles. Iso, depth of field and shutter speed were optimized by trial and error to provide the best possible videos. Another $\mathrm{GH} 5$ camera placed in front of the silicone model had different settings to capture faster emboli: ISO 640, F10 and shutter 1/3200s were used. The lighting was controlled via two side-LED panels placed on both sides of the silicone block and a frontal angled panel to avoid direct light into the lens. Two led panels (top and bottom) were also used in the mentioned tube to enhance particles contrast and therefore detection. This method allowed for counting how many particles effectively entered the ascending aorta.

\section{Post-Treatment and Video Analysis}

The videos were analyzed using a Python script with the OpenCV library. Background subtraction was performed on each frame followed by a color analysis of the pixels to differentiate particles from parasitic bubbles. The python script was used to detect and record the position of the center of mass of any particle at any given frame (a particle was defined by a pixel area range and RGB values relating to green). Then, a MATLAB script was used to analyze the recorded trajectories and to detect the particles 
crossing through each BCA, LCCA, LSA and AAO sections. This method allowed for multiple crossings within the same frame as well as backwards crossings to be accounted.

\section{Results And Discussion}

\section{Algorithm Validation}

At first, the algorithm has been tested and validated on small sets of particles with visual double-check performed manually. 10 batches of 20-40 particles have been injected and the algorithm mentioned above was used to count them. The two extreme sizes were used for validation purposes: 250-325 microns and 850-1000 microns. The pulsatile flow rate conditions were used for this validation. For 850 microns no detection errors were made, i.e. all particles detected at each specific time have been confirmed by visual inspection by 2 independent operators. Only 2\% percent of the 10 batches of 250 microns particles have been missed either because the particles were trapped in an air bubble or due to the lack of image contrast from the background for particles travelling too fast.

\section{Repartition of Particles for the Non-Pathological Signal}

The experiments were performed as follows: for each particle size, 4 sets of 200-250 particles were injected into the test-bed under pulsatile flow conditions. The number of particles needed to be injected was determined by continuous analysis until the proportions became stable. Each video was analyzed with the Python and Matlab scripts (Fig. 5) and proportions were established from Excel processing. Variability between experiments was evaluated and is available in Supplementary Tables 1 and 2.

For each model, polyethylene particles were injected to constitute an experiment in the form of 4 sets of 200-250 of each particle size. The pulsatile flowrate was maintained throughout the tests. Then thrombi made from human bloods were injected into the model constituting 4 sets of 200 clots to verify if the choice of particle density had a significant impact.

The percentages displayed in Supplementary Table 1 and 2 are plotted respectively in Fig. 6 and Fig. 7 for the stroke patient model (model 1) and the healthy patient (model 2).

\section{Discussion}

For each particle size, the statistical repartition of emboli followed the same trend. The pump percentage is the result of averaging across consecutive flowrate periods. From Fig. 6 and Fig. 7, one can point the fact that, from the statistical dispersion range, smaller-sized particles follow the flowrate repartition more closely. It can be explained by the fact that they have less inertia and are more easily driven by the flow stream. The opposite seems to hold true for larger emboli. The 850 microns particles are more often found in the upper arteries as, by their inertia, they are less able to follow the flow curvature in the aortic arch. Sets of human thrombi were found to follow the same trends as the artificial thrombi, although having various sizes ranging from 1 to $2 \mathrm{~mm}$ in size from visual inspection. 
Emboli repartitions from several hundreds of particles were evaluated for both geometries and the data did not allow to discern between the two models. Data from these in-vitro experimental results indicates that, statistically, the emboli distribution is close to the flowrate repartition in the two tested silicone model geometries. This experimental result provides insight that the aortic-arch geometry and the diameter of the arteries can directly affect the probability that an embolus can enter an artery since flowrate repartition will change.

In contrast to other emboli repartition studies, where AF and $\mathrm{N}$ flowrates are used to make a distinction between stroke propensity [2], [3], our results indicate that in the presence of clots, even under normal cardiac conditions, emboli are still able to easily exit from arteries leading to the brain, in proportion to the flowrate. For the Circle of Willis, Chung 6 concludes that smaller emboli are carried proportionally to the flow volume which seems to be confirmed by the present study. A change of model morphology from the first model to the second one does not change trends considering experimental error. Larger emboli (850 microns) were found to have, in both models, less frequency to exit through the descending aorta which can be a consequence of the models being in a vertical position, or that these emboli have more inertia and possess a lesser chance of having their initial trajectory deflected.

These results are to be put in perspective with the modeling limitations, namely that the in-vitro experiments were performed in a controlled environment using distilled water and spherically size-gauged particles. Although simplifying the fluid dynamics, the level of detail reached by imaging provides fundamental aspects of particle (and embolus) dynamics within a patient-based aortic-arch phantom.

\section{Conclusions}

In-vitro experiments of emboli dynamics in patient-based silicone aortic-arch phantoms have been made on a novel test-bed placed in a standing-patient configuration. Spherical polyethylene particles serving as thrombi analogues were injected before the ascending aorta as well as thrombi made from human blood. Image analysis software was used to count and determine the emboli statistic repartitions across the 4 arteries (BCA, LCCA, LSA and DAO). Results indicate that the repartitions of particles and blood thrombi into the aortic arch branches closely follow the flowrate repartitions suggesting that the morphology of the arteries has a primordial impact on the number of clots which can be found in arteries and can potentially provoke stroke. More research is needed in the future to verify this assertion, namely using different pulsatile flow conditions or other liquids such as artificial blood.

\section{Abbreviations}

AAO: Ascending Aorta

DAO: Descending Aorta

BCA: Brachio-Cephalic Artery 
LCCA: Left Common Carotid Artery

LSA: Left Subclavian Artery

HBC: Human Blood Clot

AF: Atrial Fibrillation

\section{Declarations}

\section{Sources of Funding}

The authors acknowledge the Région Wallonne (Belgium) for funding under Convention $\mathrm{N}^{\circ} 7463$. The authors declare no competing interests.

\section{Acknowledgements}

M.B. wrote the manuscript and was responsible for the python software, imaging, analysis of the data. M.T. has been responsible for the test-bed operation including pump programming and setup, injection of particles, Matlab programming. M.T. and M.B. both design the experiments and the test-bed. K.Z. provided the human thrombi, A.V provided the MRI datasets and the hemodynamic data. G.C., L.G. and H.F. supervised the study. All authors reviewed the manuscript.

\section{Data Availability}

All data generated or analyzed during this study are included in this published article (and its Supplementary Information files).

\section{References}


1. Feigin, V. L., Global, regional, and national burden of neurological disorders during 1990-2015: a systematic analysis for the Global Burden of Disease Study 2015. Lancet Neurology, 877-897 (2017).

2. Malone, F. et al., Investigation of the Hemodynamics Influencing Emboli Trajectories Through a Patient-Specific Aortic Arch Model. Stroke, 1531-1538 (2019).

3. Choi, H. W., Luo, T., Navia, J. A. \& Kassab, G. S., Role of Aortic Geometry on Stroke Propensity based on Simulations of Patient-Specific Models. Plos One, 0073485 (2013).

4. Fabbri, D., Lon, Q., Das, S. \& Pinelli, M., Computational modelling of emboli travel trajectories in cerebral. Biomech Model Mechanobiol 13, 289-302 (2014).

5. Mukherjee, D., Padilla, J. \& Shadden, S. C., Numerical investigation of fluid-particle interactions for embolic stroke. Theoretical and Computational Fluid Dynamics 30, 23-39 (2016).

6. Chung, E. et al., Embolus Trajectory Through a Physical Replica of the Major Cerebral Arteries. Stroke 41 (4), 647-652 (2010).

7. Malone, F. et al., Embolus Analog Trajectory Paths Under Physiological Flowrates Through Patient-Specific Aortic Arch Models. Journal of Biomechanical Engineering, 101007-1-11 (2019).

8. Itoh, Y. \& Suzuki, N., Control of brain capillary blood flow (32(7)), 1167-1176 (2012).

9. Carr, I., Nemoto, N., Schwartz, R. S. \& Shadden, S., Size-dependent predilections of cardiogenic embolic transport (305), H732-H739 (2013).

10. Malone, F. et al., An in vitro assessment of atrial fibrillation flow types on cardiogenic emboli trajectory paths. Proceedings of the Institution of Mechanical Engineers Part H Journal of Engineering in Medicine (234(12)), 1421-1431 (2020).

11. Alastruey, J., Xiao, N., Fok, H., Schaeffter, T. \& Figueroa, C. A., On the impact of modelling assumptions in multi-scale, subject-specific models of aortic haemodynamics. J. R. Soc. Interface 13: 20160073 (2016).

12. Bushi, D. et al., Hemodynamic Evaluation of Embolic Trajectory in an Arterial Bifurcation: An InVitro Experimental Model. Stroke, 2696-2700 (2005).

13. Hirshkowitz, M. e. a., National Sleep Foundation's updated sleep duration recommendations: final report, 233-243 (2015).

14. Chodzynski, K., Coussement, G. \& Zouaoui-Boudjeltia, K., France, Netherlands, Belgium, Luxemburg Patent No. EP2779144 (2014).

15. Kikinis, R., Pieper, S. \& Vosburgh, K., 3D Slicer: a platform for subject-specific image analysis, visualization, and clinical support. Intraoperative Imaging Image-Guided Therapy (2014).

16. Cutnell, J. \& Kenneth, J., Physics Fourth Edition (Wiley, 1998).

17. Guyton, A. C. \& Hall, J. E., Textbook of medical physiology (Elsevier Science, 2005).

18. Beldi, G., Beng, L., Siegel, G., Bisch-Knaden, S. \& Candinas, D., Prevention of perioperative thromboembolism in patients with atrial fibrillation. British Journal of Surgery, 1351-1355 (2007).

\section{Figures}




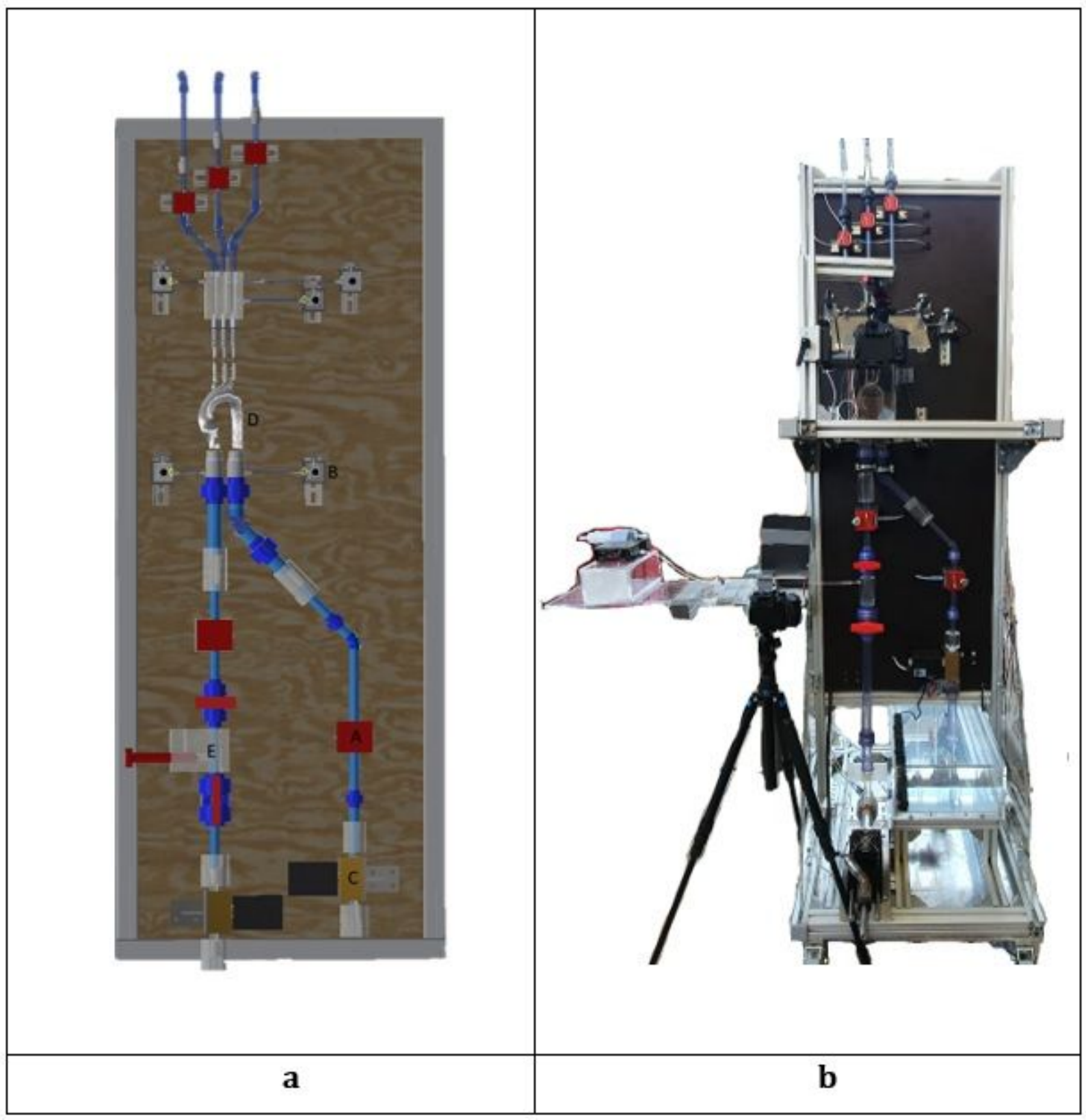

Figure 1

a Schematic front view of the in-vitro test-bed and instruments chain. A: flowmeter, B: pressure sensor, C: solenoid valve, D: aortic-arch model, E: injection site. b Front view photography of the in-vitro test-bed. 


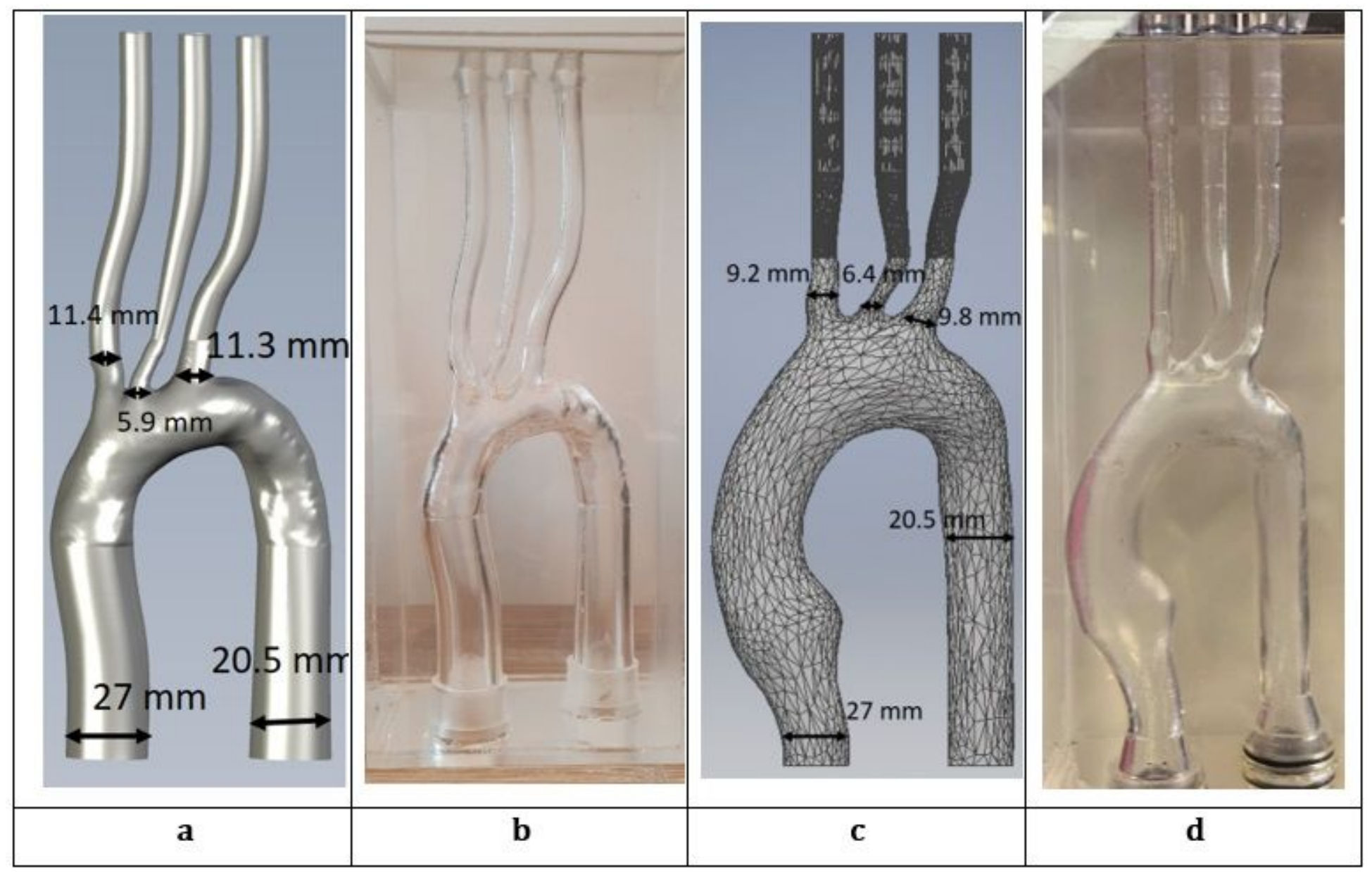

Figure 2

a STL geometry obtained from 3D Slicer and Inventor modifications for model 1. b Silicone block upon dissolution of the model's 3D pva print. c STL for model 2 and. $d$ Associated silicone block 
Geometry adapted flowrate and testbed reproduction

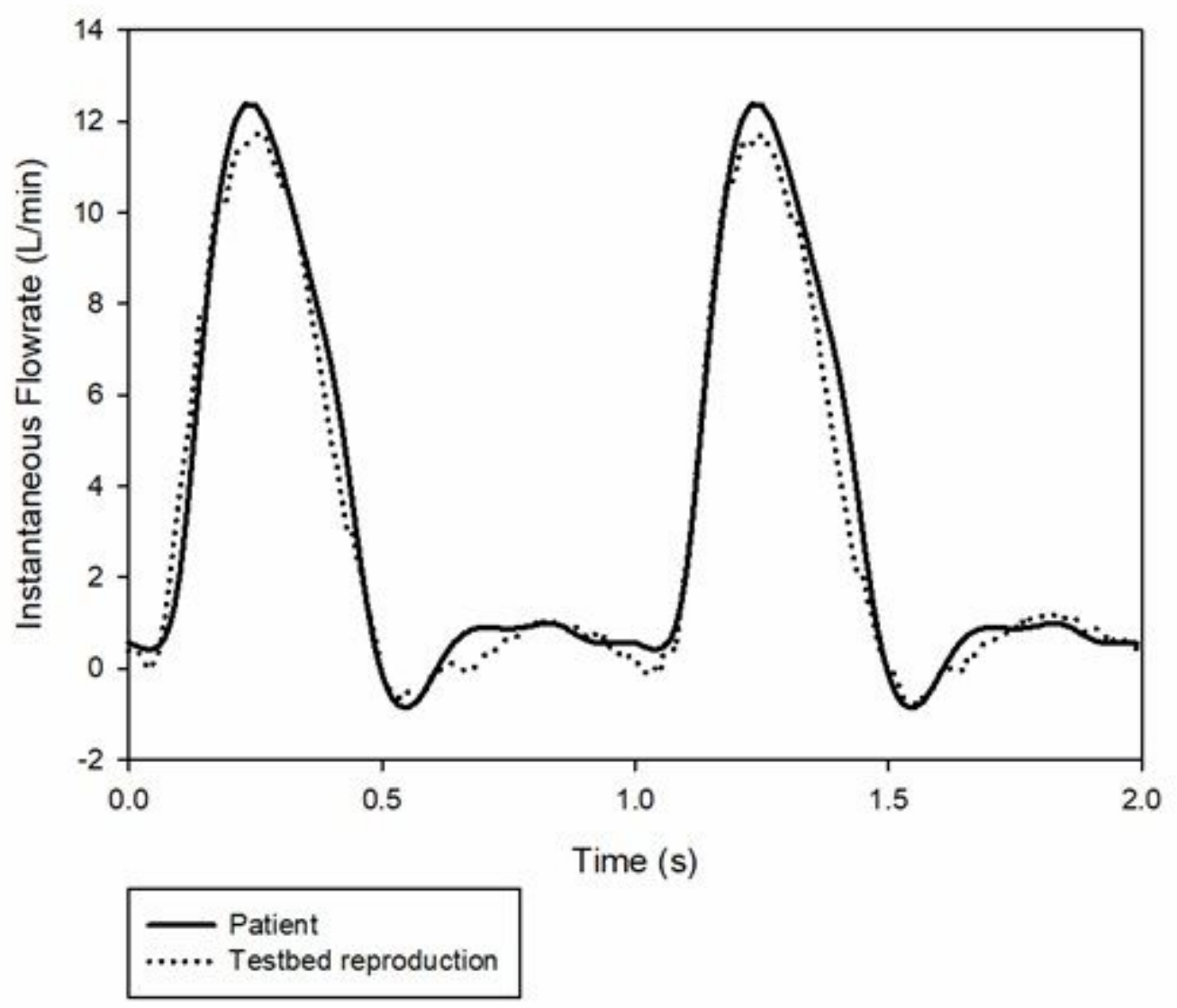

Figure 3

Adapted patient flowrate for the model geometry to keep the speed constant (solid line) as well as the flowrate reproduction by the test-bed (dotted line). 
10 second excerpt of the flowrates measured by the testbed

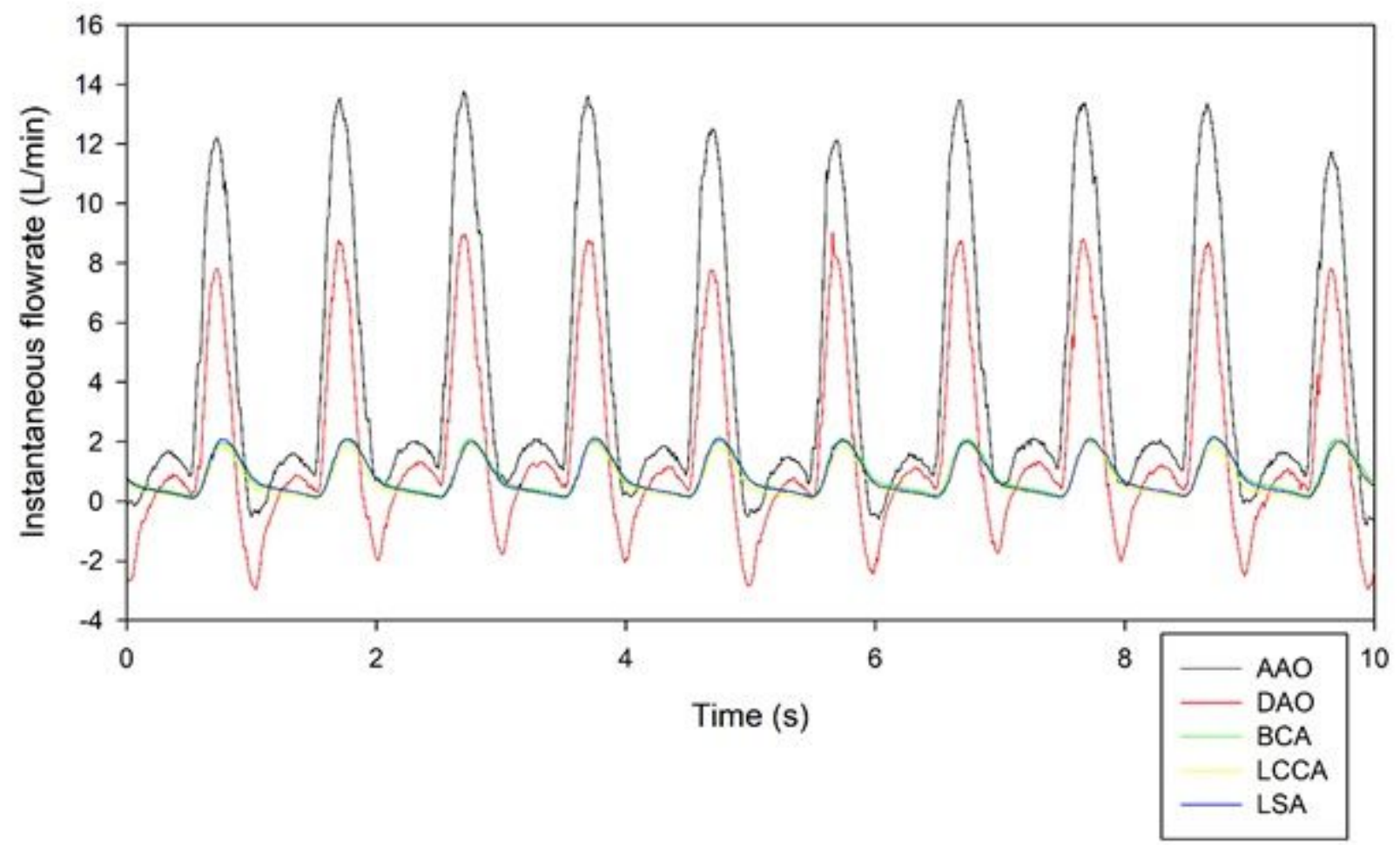

Figure 4

Instantaneous pulsatile flowrates for the 5 branches of Model 1. AAO (black line), DAO (red line), BCA (green), LCCA (yellow) and LSA (Blue) cycles for ten seconds of continuous test-bed operation. 


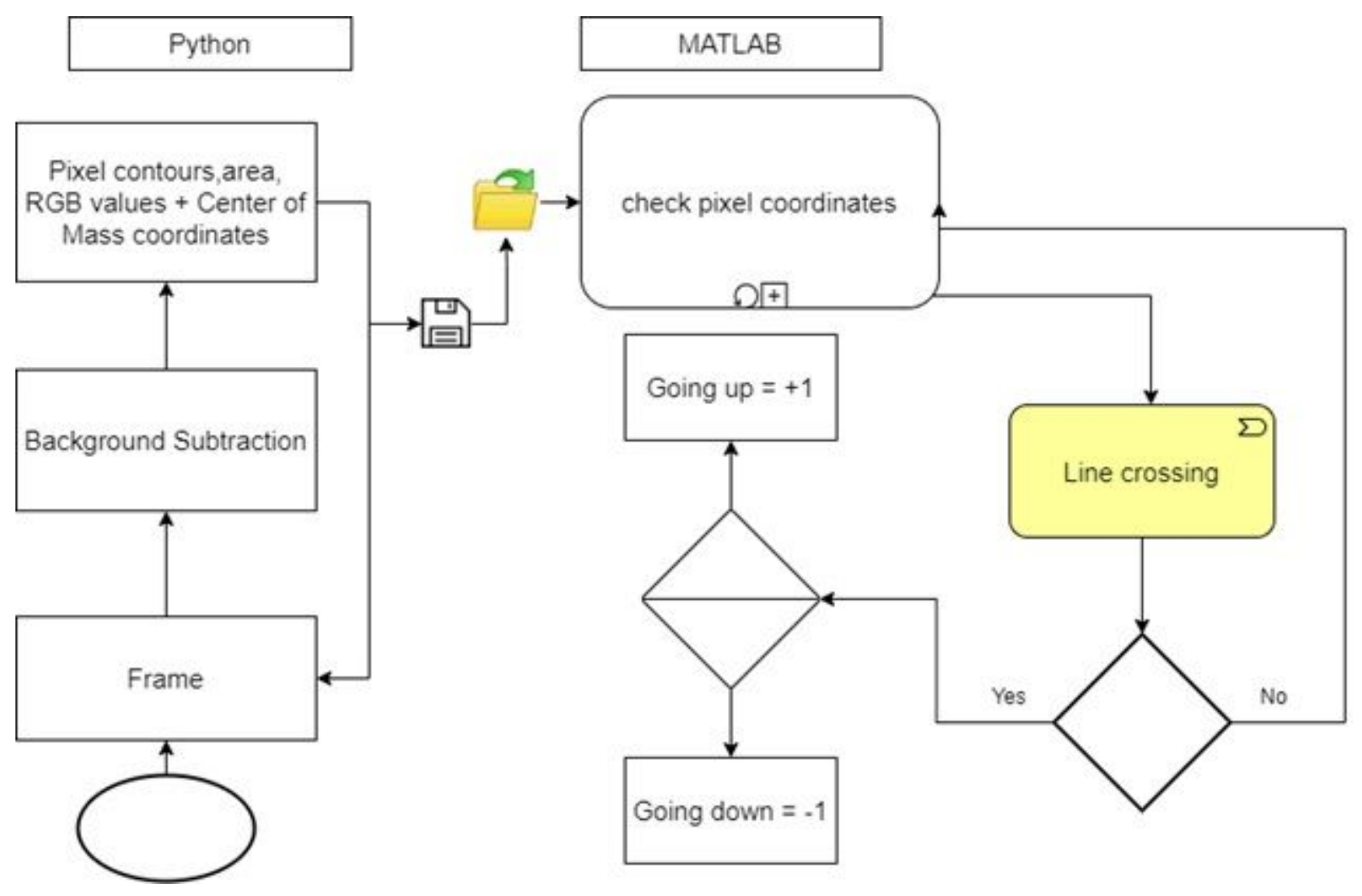

Figure 5

Algorithm flowchart. On the left, the Python flowchart for which data is gathered. Background subtraction is made on each frame to obtain a mask. For each detection, the pixels' center of mass is saved and the RGB pixel value. On the right the same data is used by Matlab to count the emboli. 
REPARTITION PERCENTAGES OF ARTIFICIAL THROMBI, HUMAN BLOOD CLOTS AND PUMP FLOWRATE PERCENTAGES, Model 1

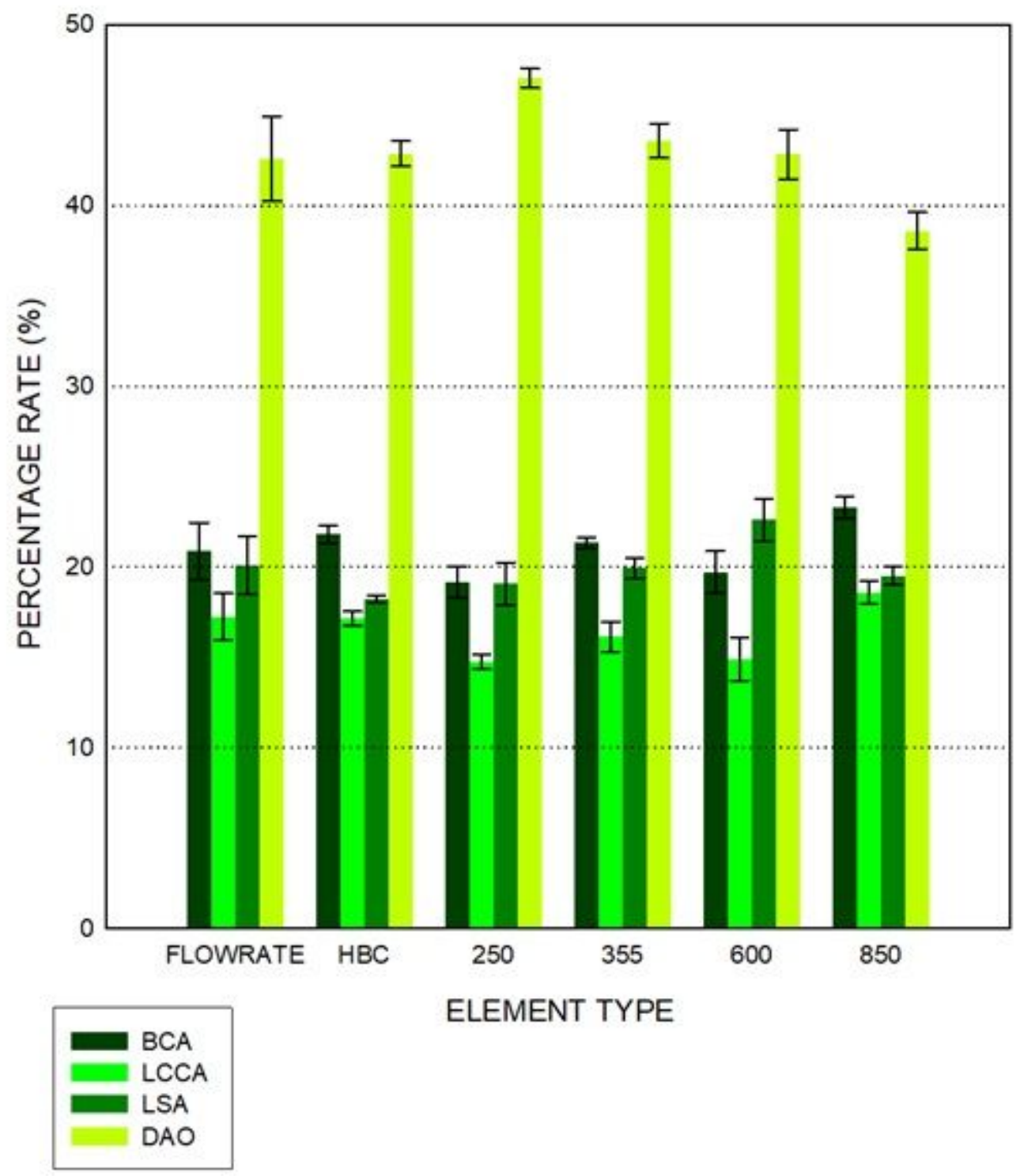

Figure 6

Repartition percentages for the different types of $\mathrm{N}$ injected particles, for $250 \mu \mathrm{m}$ the total number of injected particles $\mathrm{N}=845$; for $355 \mu \mathrm{m} \mathrm{N}=1275$; for $600 \mu \mathrm{m}, \mathrm{N}=969$; for $850 \mu \mathrm{m}, \mathrm{N}=1185$ and for human blood clots, $\mathrm{N}=1197$. Each set of bars represent the repartition percentages with corresponding error calculated in each case as the variability for each experiment. For each artery with the pump flowrate associated percentage in the following order: in dark green, brachiocephalic artery; in lime green, left common carotid artery; in green, left subclavian artery and in fluo grey the descending aorta. 


\section{REPARTITION PERCENTAGE RATES OF ARTIFICIAL THROMBI AND PUMP FLOWRATE PERCENTAGE RATES, Model 2}

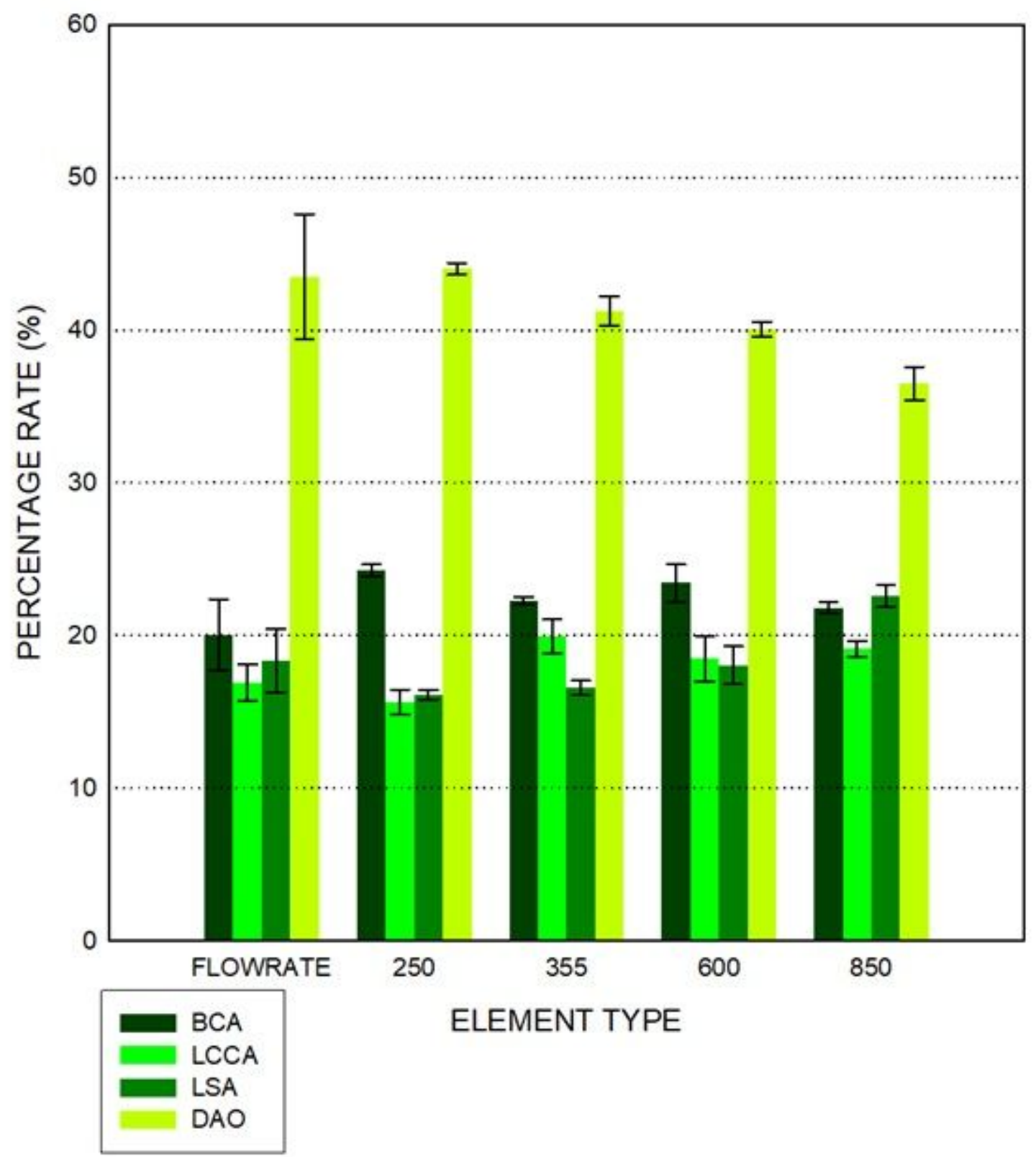

Figure 7

Repartition percentages for the different types of $\mathrm{N}$ injected particles, for $250 \mu \mathrm{m}$ the total number of injected particles $\mathrm{N}=929$; for $355 \mu \mathrm{m} \mathrm{N}=973$; for $600 \mu \mathrm{m}, \mathrm{N}=726$; for $850 \mu \mathrm{m}, \mathrm{N}=608$. Each set of bars represent the repartition percentages with corresponding error calculated in each case as the variability for each experiment. For each artery with the pump flowrate associated percentage in the following order: in dark green, brachiocephalic artery; in lime green, left common carotid artery; in green, left subclavian artery and in fluo grey the descending aorta. 


\section{Supplementary Files}

This is a list of supplementary files associated with this preprint. Click to download.

- supplementarydata.docx 